\title{
NORMAL AND INVERTED ALGAN/GAN BASED PIEZOELECTRIC FIELD EFFECT TRANSISTORS GROWN BY PLASMA INDUCED MOLECULAR BEAM EPITAXY
}

\author{
M. J. Murphy ${ }^{\mathrm{a}}$, B. E. Foutz ${ }^{\mathrm{a}}$, K. Chu ${ }^{\mathrm{a}}$, H. Wu ${ }^{\mathrm{a}}$, W. Yeo ${ }^{\mathrm{a}}$, W. J. Schaff, O. Ambacher ${ }^{\mathrm{a}}$,

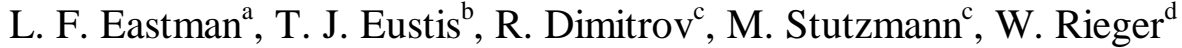 \\ ${ }^{\text {a }}$ School of Electrical Engineering, Cornell University, Ithaca, NY 14853 \\ ${ }^{\mathrm{b}}$ Department of Materials Science, Cornell University, Ithaca, NY 14853 \\ ${ }^{c}$ Walter Schottky Institute, TU-Munich, Am Coulombwall, D-85748 Garching, Germany \\ ${ }^{\mathrm{d}}$ Ferdinand Braun Institute, Rudower Chaussee 5, D-12489 Berlin, Germany
}

Cite this article as: MRS Internet J. Nitride Semicond. Res. 4S1, G8.4(1999)

\begin{abstract}
High quality Ga-face and $\mathrm{N}$-face $\mathrm{AlGaN} / \mathrm{GaN}$ based heterostructures have been grown by plasma induced molecular beam epitaxy. By using Ga-face material we are able to fabricate conventional heterojunction field effect transistors. Because the $\mathrm{N}$-face material confines electrons at a different heterojunction, the resulting transistors are called inverted. The Ga-face structures use a high temperature AlN nucleation layer to establish the polarity. Structures from these materials, relying only on polarization induced interface charge effects to create the two-dimensional electron gases, are used to confirm the polarity of the material as well as test the electrical properties of the layers. The resulting sheet concentrations of the two dimensional electron gases agree very well with the piezoelectric theory for this materials system. Hall mobilities of the twodimensional gases for the $\mathrm{N}$-face structures are as high as $1150 \mathrm{~cm}^{2} / \mathrm{Vs}$ and $3440 \mathrm{~cm}^{2} / \mathrm{Vs}$ for 300 $\mathrm{K}$ and $77 \mathrm{~K}$ respectively, while the $\mathrm{Ga}$-face structures yield room temperature mobilities of 1190 $\mathrm{cm}^{2} /$ Vs. Both structures were then fabricated into transistors and characterized. The inverted transistors, which were fabricated from the $\mathrm{N}$-face material, yielded a maximum transconductance of $130 \mathrm{mS} / \mathrm{mm}$ and a current density of $905 \mathrm{~mA} / \mathrm{mm}$. Microwave measurements gave an $\mathrm{f}_{\mathrm{t}}$ of 7 $\mathrm{GHz}$ and an $\mathrm{f}_{\max }$ of $12 \mathrm{GHz}$ for a gate length of $1 \mu \mathrm{m}$. The normal transistors, fabricated from the Ga-face material, produced a maximum transconductance of $247 \mathrm{mS} / \mathrm{mm}$ and a current density of $938 \mathrm{~mA} / \mathrm{mm}$. Microwave measurements gave an $\mathrm{f}_{\mathrm{t}}$ of $50 \mathrm{GHz}$ and an $\mathrm{f}_{\max }$ of $97 \mathrm{GHz}$ for a gate length of $0.25 \mu \mathrm{m}$. This shows that using plasma induced molecular beam epitaxy $\mathrm{N}$-face and $\mathrm{Ga}(\mathrm{Al})$-face $\mathrm{AlGaN} / \mathrm{GaN}$ heterostructures can be grown with structural and electrical properties very suitable for high power field effect transistors.
\end{abstract}

\section{INTRODUCTION}

The polarity of group III-nitrides is a subject that has seen much research recently. ${ }^{1}$ The fact that the material has wurtzite crystal symmetry means that there exists a polarity in the growth direction, yielding either Ga-face (0001) or N-face (0001) material. This polarity issue has several important consequences, some of which are the differing materials properties of the different polarities as well as how the different polarities can be achieved from a growth point of view. The polarity also determines the orientation of the spontaneous and piezoelectric polarization inside the material, which has drastic effects on device design. The devices under study are piezoelectric HFETs grown by Plasma Induced Molecular Beam Epitaxy (PIMBE). The transistor structures are intentionally undoped and therefore rely on polarization induced interface charge effects to create a two-dimensional electron gas (2DEG). Depending on the 
polarity of the material grown, the 2 DEG will be confined at different interfaces. Thus by determining at which interface the electrons are confined, it is possible to probe the polarity of the layers grown. These structures are also useful for comparing the electrical quality of the different polarities. We will show that high quality $\mathrm{N}$-face epitaxial layers can be deposited that are suitable for inverted transistors which can be used in high frequency applications. We will further show that PIMBE Ga-face HFETs can achieve state-of-the-art frequency response as well as excellent DC results.

\section{EXPERIMENTAL DETAILS}

The structures in this study consist of GaN/AlGaN/GaN heterostructures deposited by PIMBE. Two separate MBE machines were used to obtain this material. The N-face material, which was used to achieve the inverted HFETs, was deposited in a Tectra MBE, which uses conventional effusion cells for the Group IIIs. This system is equipped with an Oxford Applied Research CARS $25 \mathrm{RF}$ plasma source. A more detailed description of the machine and the typical growth conditions can be found in Reference 2. The transistor structure using the N-face material is unintentionally n-type, therefore a $2.5 \mu \mathrm{m} \mathrm{Mg}$ doped buffer layer is employed. By using this compensation technique, a background doping concentration in the $10^{12} \mathrm{~cm}^{-3}$ range is achievable as measured by capacitance voltage profiling and Hall measurements. $A 60 \mathrm{~nm} \mathrm{Al}{ }_{.28} \mathrm{Ga}_{.72} \mathrm{~N}$ layer is then deposited, followed by a $30 \mathrm{~nm} \mathrm{GaN}$ top layer.

The Ga-faced layers used for conventional HFETs in this research were deposited in a turbo-pumped Varian GenII MBE which also uses conventional effusion cells for Ga and Al. The nitrogen radicals for this machine are supplied via an EPI Uni-Bulb RF plasma source. A more detailed description of the machine and the typical growth conditions can be found in Reference 3 . The Ga-face device consists of a nominally $7 \mathrm{~nm}$ AlN nucleation layer, followed by a $1.2 \mu \mathrm{m} \mathrm{GaN}$ buffer layer. Unlike the aforementioned structure, this buffer layer has a background doping concentration in the $10^{12} \mathrm{~cm}^{-3}$ range, so no compensation is needed. This buffer layer is followed by a $20 \mathrm{~nm} \mathrm{Al}{ }_{.2} \mathrm{Ga}_{8} \mathrm{~N}$ layer and a $2 \mathrm{~nm} \mathrm{GaN}$ cap layer. It should be noted that the only way Gaface material was achievable was through the use of the AlN nucleation layer.

Capacitance voltage profiling is used to probe the free carrier profiles of the materials grown. This profiling was done using an HP 4275A multi-frequency LCR meter and a standard mercury probe. The normal biasing range is from 0.4 to $-10.0 \mathrm{~V}$ with 200 steps and a small signal frequency of $10 \mathrm{kHz}$ is used.

\section{THEORY}

The group III-nitrides commonly occur in the wurtzite crystal structure and lack inversion symmetry along the c-axis. This asymmetry allows for the possibility of a built-in spontaneous polarization directed along the c-axis. Calculations have shown that the group III-nitrides have a strong spontaneous polarization. ${ }^{4}$ In the case of $\mathrm{GaN}$, the direction of polarization points from the $\mathrm{N}$ atom to the $\mathrm{Ga}$ atom along the c-axis bond. As shown in Figure 1, N-faced material corresponds to $\mathrm{N}$ in the upper position of the (0001) bi-layer and the direction of the spontaneous polarization points toward the surface, opposite the (0001) direction. In the case of Ga-faced material, Ga occupies the top position of the (0001) bi-layer. In this case, the spontaneous polarization points toward the substrate. Strain perpendicular to the c-axis deforms the crystal and modifies the polarization. This is the piezoelectric effect. Details of the calculation of the piezoelectric polarization and induced sheet charges can be found in Reference 5. To determine the total polarization of a given epi-layer the spontaneous polarization of the relaxed material is 
added to the change in polarization due to the strain. A more complete treatment of our calculations including the spontaneous polarization will be found in Reference 6 .

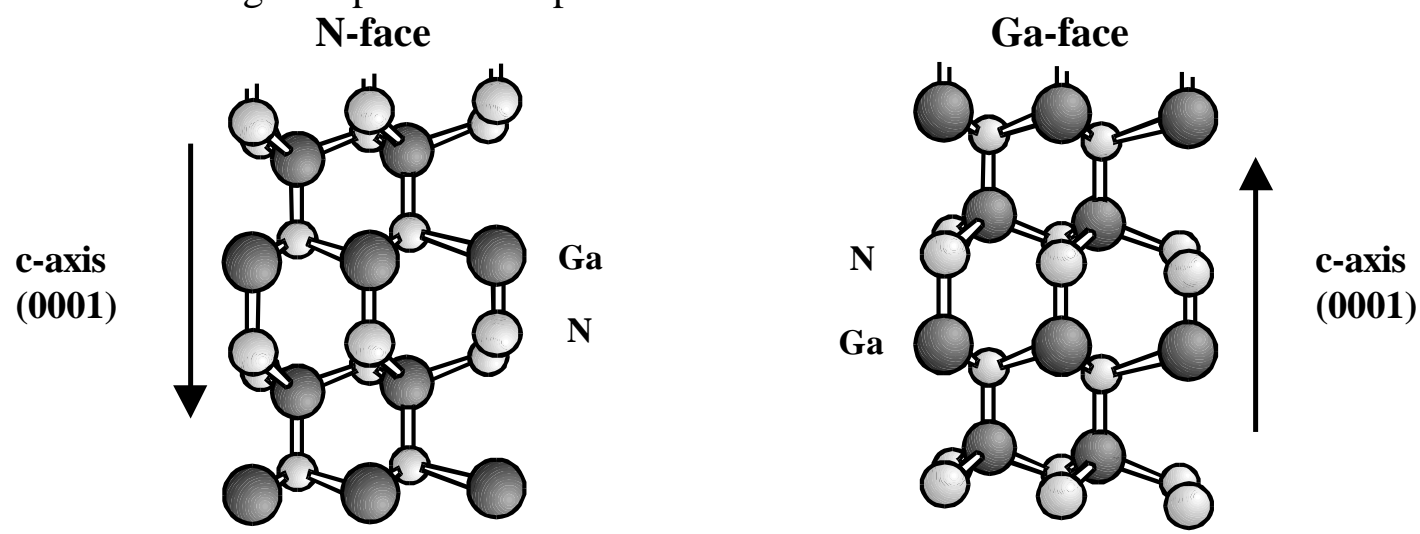

Figure 1. Schematic of the wurtzite crystal structure for the different polarities of GaN, showing the placement of the $\mathrm{Ga}$ and $\mathrm{N}$ atoms.

The polarity of the device structure, $\mathrm{N}$-face or Ga-face, determines where a 2DEG will form. As can be seen in Figure 2, the total polarization $(\mathbf{P})$ in the $\mathrm{AlGaN}$ is flipped for the case of differing material polarity. The spontaneous polarization of the relaxed $\mathrm{GaN}$ layers points in the same direction as the AlGaN layer, but is weaker. The large change in polarization from one material to the other creates a positive bound charge $\left(\sigma_{b}=\mathbf{P}_{\mathrm{AlGaN}}-\mathbf{P}_{\mathrm{GaN}}\right)$ at one of the interfaces. This charge creates a strong electric field at the interface that confines electrons and a 2DEG will form. For the case of the Ga-faced material, one expects the positive bound charge and the 2DEG to be located at the lower $\mathrm{AlGaN} / \mathrm{GaN}$ interface. This is the conventional HFET structure and is referred to as a "normal" structure. In contrast, the $\mathrm{N}$-face material will confine the 2DEG at the upper GaN/AlGaN interface, and is referred to as an "inverted" structure. Since the location of the 2DEG can be determined through the capacitance-voltage profiling technique, the polarity of the material can be determined.

\section{RESULTS AND DISCUSSION}

While two separate machines were used to obtain the different materials, it should be noted that both machines were able to obtain $\mathrm{N}$-face material without the use of the AlN nucleation layer. The superior quality of the $\mathrm{N}$-face material obtained from the Tectra MBE was more suitable for devices however. It should also be noted that recent growths from the Tectra MBE confirm the ability of the AIN nucleation layer to switch the polarity of the films deposited, and similar material properties were measured on Ga-face material from both machines.

The first structure to be studied was the inverted HFET. The measured Hall mobility for this device was $1150 \mathrm{~cm}^{2} / \mathrm{Vs}$ at $300 \mathrm{~K}$ and $3440 \mathrm{~cm}^{2} / \mathrm{Vs}$ at $77 \mathrm{~K}$ with a constant sheet density of $6.0 \times 10^{12} \mathrm{~cm}^{-2}$. The existence and position of the $2 \mathrm{DEG}$ was verified using capacitance voltage profiling. As can be seen in Figure 3, the position of the 2DEG is at the upper heterointerface, which agrees with the piezoelectric theory for $\mathrm{N}$-face material. Inverted HFETs were then fabricated from this layer structure and tested. DC measurements on devices with a gate length of $1.0 \mu \mathrm{m}$ and a gate width of $40 \mu \mathrm{m}$ yielded a maximum transconductance of $130 \mathrm{mS} / \mathrm{mm}$ and a peak current density of $905 \mathrm{~mA} / \mathrm{mm}$. Frequency tests were then performed on the devices, from which an $\mathrm{f}_{\mathrm{t}}$ of $7 \mathrm{GHz}$ and an $\mathrm{f}_{\max }$ of $12 \mathrm{GHz}$ were obtained. 


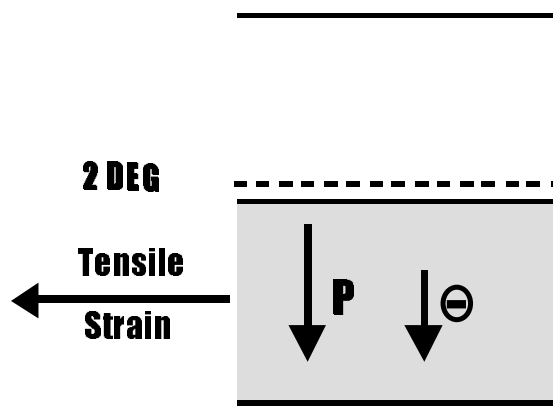

"Inverted"
AGall

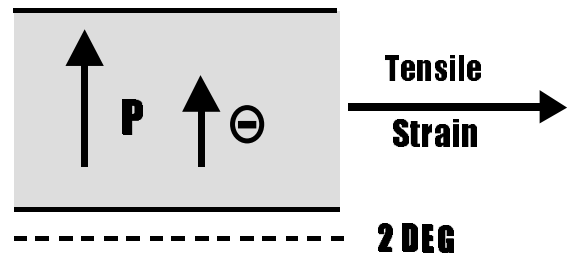

Gall

Figure 2. Schematic layer structure for $\mathrm{GaN} / \mathrm{AlGaN} / \mathrm{GaN}$ heterostructures showing both the total polarization $(\mathbf{P})$ in the $\mathrm{AlGaN}$ layer and the interfaces at which the $2 \mathrm{DEG}$ will be confined. The $\mathrm{N}$-face yields a 2DEG at the upper interface and hence is called an inverted structure, while the Ga-face confines carriers at the bottom interface and is called a normal structure.

The Ga-face material under study gave a Hall mobility of $1190 \mathrm{~cm}^{2} / \mathrm{Vs}$ at $300 \mathrm{~K}$, with a sheet density of $1.0 \times 10^{13} \mathrm{~cm}^{-2}$. Results of capacitance voltage profiling of this structure are shown in Figure 4. This data proves the existence and position of a $2 \mathrm{DEG}$, only this time the confinement is at the lower heterointerface, which agrees with the piezoelectric theory for Ga-face material. Conventional HFETs were then fabricated from this material and tested, the results appear in Figure 5. DC measurements on devices with a gate length of $0.25 \mu \mathrm{m}$ and a gate width of 100 $\mu \mathrm{m}$ yielded a maximum transconductance of $247 \mathrm{mS} / \mathrm{mm}$ and a peak current density of 938 $\mathrm{mA} / \mathrm{mm}$. With a $-6 \mathrm{~V}$ bias applied to the gate contact, breakdown voltages as high as $60 \mathrm{~V}$ from source to drain were attained. Microwave measurements were then performed on the devices, from which an $\mathrm{f}_{\mathrm{t}}$ of $50 \mathrm{GHz}$ and an $\mathrm{f}_{\max }$ of $97 \mathrm{GHz}$ were obtained, as shown in Figure 5, however values of $f_{t}$ as high as $60 \mathrm{GHz}$ were measured for the devices with slightly smaller gate lengths.

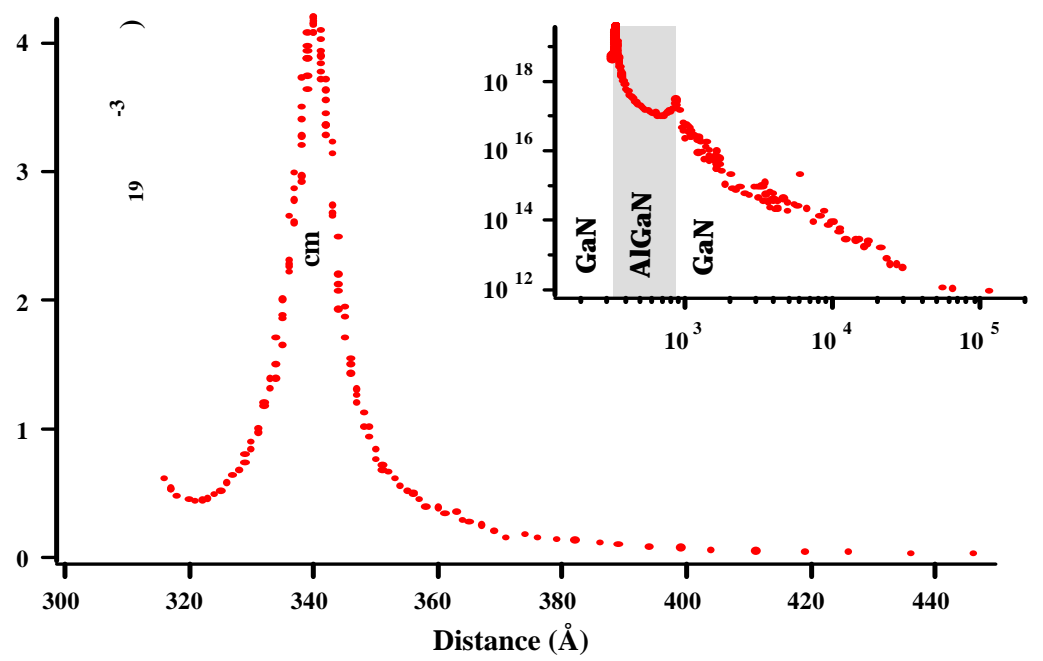


Figure 3. Capacitance voltage profile for the inverted HFET. The graphs clearly show a 2DEG that is confined at the upper interface. This agrees perfectly with the piezoelectric theory for $\mathrm{N}$ face material.

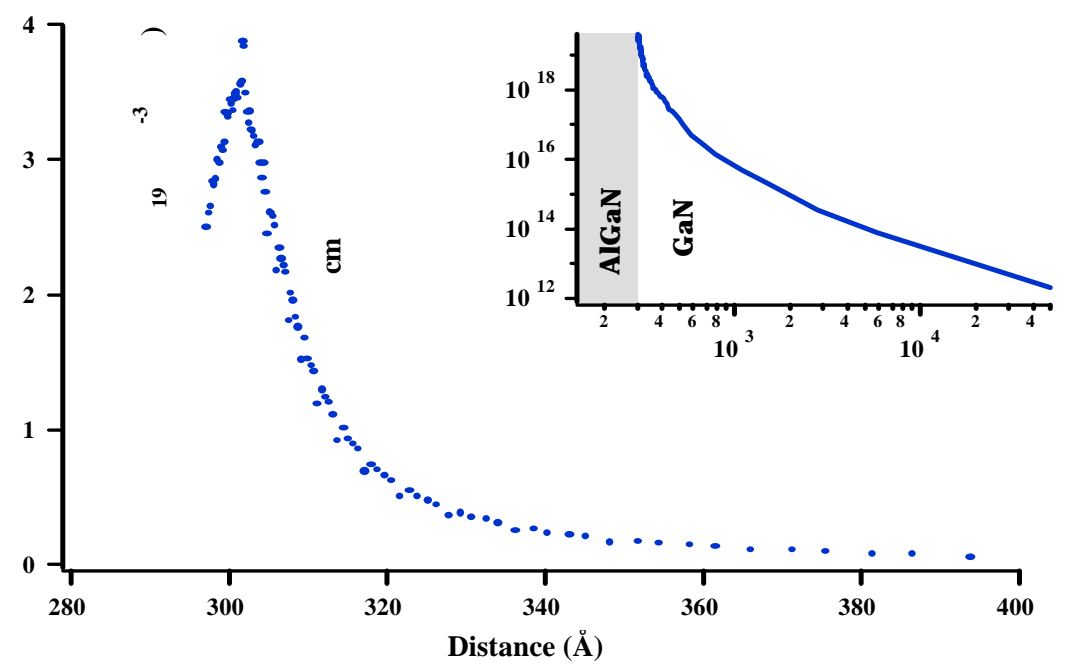

Figure 4. Capacitance voltage profile for the normal HFET. The graphs clearly show a 2DEG that is confined at the lower interface. This agrees perfectly with the piezoelectric theory for Gaface material.

\section{CONCLUSION}

Several important issues have been covered in this research. Firstly, it has been shown that capacitance voltage profiling can be used as a tool to determine the polarity of the GaN material in question by determining at which interface the $2 \mathrm{DEG}$ is confined. Secondly, it has been shown that piezoelectric HFETs are possible in both polarities of (Al)GaN material. Both the $\mathrm{N}$-face and $\mathrm{Ga}$-face material appear to have a single polarity across the entire wafer and no evidence of inversion domain boundaries is seen by Hall measurements, C-V profiling, or device testing. While the $\mathrm{N}$-face material in this study is of excellent electrical quality, it is possible to attain Ga-face material with PIMBE by utilizing an AIN nucleation layer. This Ga-face material can be used to create state-of-the-art HFETs with frequency response that is comparable to the best attainable in $(\mathrm{Al}) \mathrm{GaN}$ by MOCVD. ${ }^{7}$ 

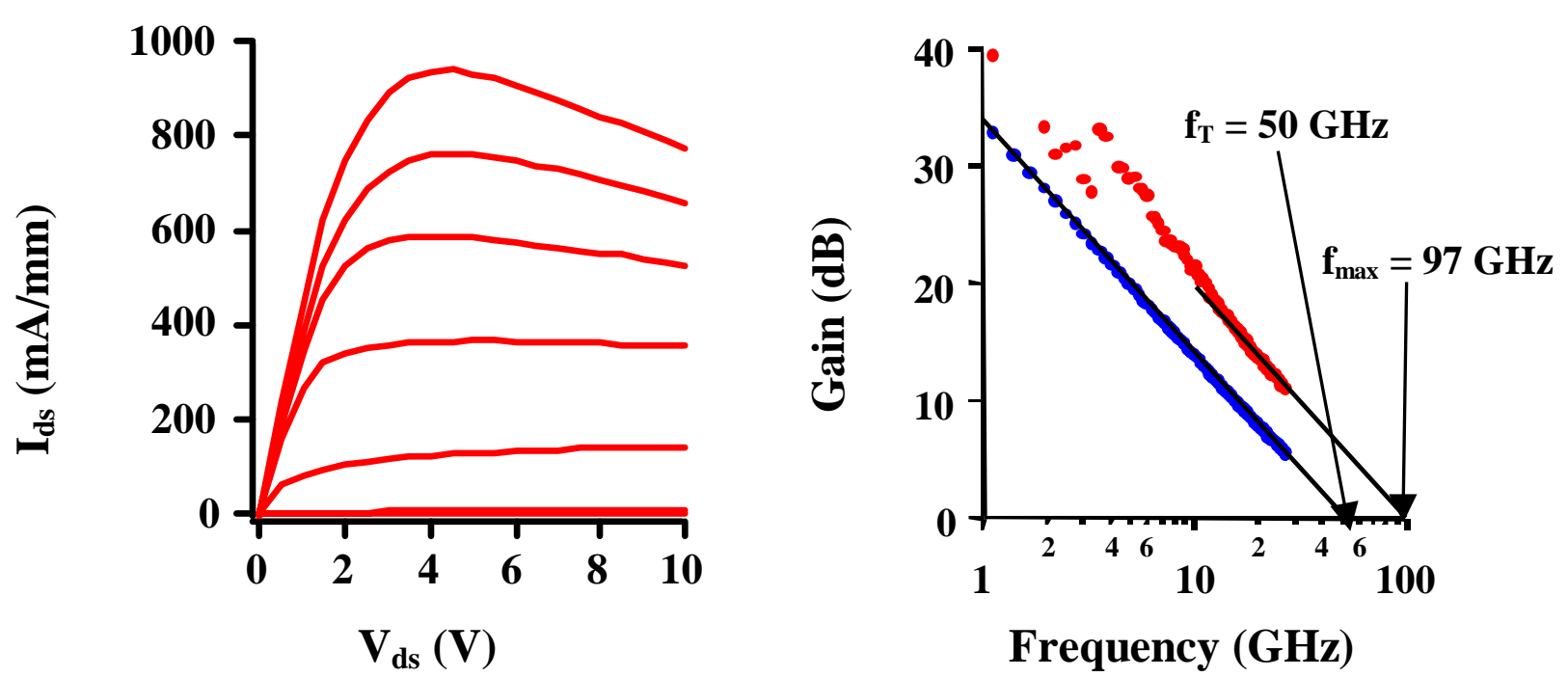

Figure 5. Results from DC and microwave measurements on a normal HFET with a gate length of $0.25 \mu \mathrm{m}$ and a gate width of $100 \mu \mathrm{m}$. For the DC curves, the gate bias starts at $+1.0 \mathrm{~V}$ and drops by $1.0 \mathrm{~V}$ for each increment.

\section{ACKNOWLEDGEMENTS}

Dr. Ambacher would like to thank the Alexander von Humboldt Stiftung for a Feodor Lynen fellowship. The work done at Cornell University is supported by the Office of Naval Research under Contracts No. N00014-96-1-1223 and N00014-95-1-0926 under the direction of Dr. John Zolper. The work done at the Walter Schottky Institute was funded by the Deutsche Forschungsgemeinschaft (Stu 139/2).

\section{REFERENCES}

${ }^{1}$ E. S. Hellman, MRS Internet J. Nitride Semicond. Res. 3, 11 (1998).

${ }^{2}$ R. Dimitrov, L. Wittmer, H. P. Felsl, A. Mitchell, O. Ambacher and M. Stutzmann, phys. stat. sol. 168, R7 (1998).

${ }^{3}$ M. J. Murphy, K. Chu, T. J. Eustis, J. Smart, H. Wu, W. Yeo, W. J. Schaff, O. Ambacher, J. R. Shealy and L. F. Eastman, "MBE growth of normal and inverted two-dimensional electron gases in AlGaN/GaN based heterostructures," in print J. Vac. Sci. Tech. B.

${ }^{4}$ F. Bernardini, V. Fiorentini, and D. Vanderbilt, Phys. Rev. B 56, 16 (1997).

${ }^{5}$ P. M. Asbeck, E. T. Yu, S. S. Lau, G. J. Sullivan, J. Van Hove, and J. Redwing, Elec. Lett. 33, 1230 (1997).

${ }^{6}$ O. Ambacher, J. Smart, J. R. Shealy, N. G. Weimann, K. Chu, M. Murphy, W. J. Schaff, and L. F. Eastman "Two dimensional electron gases induced by spontaneous and piezoelectric polarization charges in N- and Ga-face AlGaN/GaN heterostructures," in print J. Appl. Phys.

${ }^{7}$ K. K. Chu, J. Smart, J. R. Shealy, and L. F. Eastman, SOTAPOCS, 1998. 